\title{
Etablir et comparer les spectres biologiques de plusieurs groupements végétaux
}

\author{
J.-P. Aberlin ${ }^{1}$ P. Daget ${ }^{2}$
}

\section{Mots-clés}

Savane - Mesure - Végétation Ecosystème - Mali.

\section{Résumé}

La structure de la flore d'une station peut être caractérisée par son spectre biologique qui indique le taux de chacun des types biologiques définis par Raunkiaer dans la flore stationnelle. Un groupement réunissant plusieurs stations peut être caractérisé par un spectre biologique moyen. Certains des résultats de I'analyse discriminante permettent la comparaison des profils biologiques de plusieurs groupements végétaux compte tenu de leur variabilité interne : application à des groupements de savane du Mali.

\section{PRINCIPES}

\section{Le concept de types biologiques}

Reprenant une idée déjà ancienne (7), Warming définit en 1884 le concept de forme biologique pour traduire « la forme que le corps végétatif de la plante revêt en harmonie avec le milieu environnant et sous laquelle s'accomplissent les phénomènes vitaux » (13), mais la définition des formes elles-mêmes est longtemps restée confuse. C'est seulement en 1904 que les types biologiques ont été définis par l'écologue danois Raunkiaer (14) de la manière suivante :

- les phanérophytes $(\mathrm{Ph})$, pour lesquels les bourgeons végétatifs sont situés à l'extrémité de tiges ligneuses assez loin du sol. Selon la hauteur, on distingue : les macrophanérophytes (Mph), dont les

\footnotetext{
1. Laboratoire de botanique et de phytosociologie, université de Franche-Comté, 25000 Besançon, France

E-mail : jean-pierre.aberlin@univ-fcomte.fr

2. Cirad-emvt et Grefo, TA 30/E, campus international de Baillarguet,

34398 Montpellier Cedex 5, France

E-mail : philippe.daget@ciradfr
}

tiges ligneuses dépassent $8 \mathrm{~m}$ de hauteur ; les mésophanérophytes (meph), dont les tiges ligneuses ont entre 2 et $8 \mathrm{~m}$ de hauteur ; les microphanérophytes (miph), dont les tiges ligneuses ont entre 0,5 et $2 \mathrm{~m}$ de hauteur ; les nanophanérophytes ( $\mathrm{Nph})$, dont les tiges ligneuses ne dépassent pas $0,5 \mathrm{~m}$ de hauteur ;

- les chamaéphytes $(\mathrm{Ch})$, pour lesquels les bourgeons sont très voisins de la surface du sol ;

- les hémicryptophytes $(\mathrm{H})$, pour lesquels les bourgeons sont situés à la surface du sol ;

- les cryptophytes $(\mathrm{C})$, pour lesquels les bourgeons sont situés à l'intérieur du sol. Selon le type de sol, les subdivisions suivantes sont reconnues : les géophytes $(\mathrm{G})$, dont les bourgeons sont dans un sol terrestre sain ; les hélophytes (Hé), dont les bourgeons sont dans un sol terrestre très humide comme de la vase ; les hydrophytes (Hy), dont les bourgeons sont dans un sol subaquatique ;

- les thérophytes (T), qui sont les plantes de l'été ou de la saison favorable pour la végétation et qui passent la saison défavorable sous forme de graine.

L'applicabilité en régions tropicales du concept de type biologique a fait l'objet de nombreuses discussions dans le passé $(2,12)$, mais elle ne semble plus poser de problème actuellement $(8,9,11)$. 


\section{Spectres biologiques}

Ces types biologiques caractérisent les espèces ; pour le tapis végétal, Raunkiaer introduit en 1908 le concept de spectre biologique qui réunit les proportions de chacun des types biologiques dans la flore analysée, d'abord au niveau régional (17), puis aux niveaux de la station et du groupement $(16 ; 17)$. Le spectre d'une association peut être établi comme celui de la station sur l'ensemble de la flore concernée, mais aussi par l'établissement d'un spectre moyen entre les individus d'association pris en compte. C'est ce qui a été retenu ici.

L'intérêt principal des spectres biologiques est qu'ils reflètent, par la structure de la végétation dont ils sont une traduction, les conditions du milieu ambiant $(15,16,17)$, tant au niveau stationnel que régional, mais pas au niveau interrégional (5). Il est donc indispensable de pouvoir comparer rigoureusement les spectres moyens d'associations voisines et de distinguer les différences minimes non significatives des écarts qui le sont.

\section{Comparaison des spectres}

Les méthodes de l'analyse discriminante sont particulièrement adaptées à ce type de comparaison parce qu'elles n'impliquent rien quant à la structure intrinsèque des données mises en jeu (3) Si elles sont si peu employées en phytologie, c'est parce que les biométriciens qui les présentent $(4,10,20)$ les masquent trop souvent derrière un formalisme théorique utile pour eux, mais opaque pour la plupart des utilisateurs, comme l'est aussi la grande variété d'options suggérées par les notices qui accompagnent les logiciels (19). En fait, en préparant les données de manière adéquate, l'application et l'interprétation de l'analyse discriminante sont aussi simples que n'importe quelle autre méthode.

\section{APPLICATION}

Un exemple, repris de la base Flotrop à partir de données recueillies au nord-est de Bamako (Mali) (1) et traitées par les procédures du système $\operatorname{SAS}^{\odot}(6,18)$, montre le mode opératoire et la nature des résultats utiles. Il s'agit d'un ensemble de 32 relevés relatifs à cinq groupements :

- savanes primaires arbustives (savane 1)

- savanes primaires à Ctenium newtonii (savane 4)

- savanes primaires à Cordyla pinnata (savane 2)

- savanes secondaires à Diospyros mespiliformis (savane 3)

- savanes secondaires à Swartzia madagascarensis (savane 4)

Pour la préparation des données on retient, pour commencer, neuf types biologiques :

- les macrophanérophytes (A)

- les mésophanérophytes (M)

- les microphanérophytes (I)

- les nanophanérophytes $(\mathrm{N})$

- les chamaéphytes (C)

- les hémicryptophytes $(\mathrm{H})$

- les géophytes $(\mathrm{G})$

- les thérophytes (T)

- les hydrophytes $(\mathrm{O})$

Les symboles utilisés ici sont différents de ceux que Raunkiaer avait proposés, mais ils ont l'avantage de n'employer qu'un seul caractère. Ensuite, les observations de terrain sont formatées en bordereau (tableau I),

\section{Tableau I}

Spectres de chacune des quatre stations de savane primaire arbustive de la dition

\begin{tabular}{ccccccccc}
$\mathbf{A}$ & $\mathbf{M}$ & $\mathbf{I}$ & $\mathbf{N}$ & $\mathbf{C}$ & $\mathbf{H}$ & $\mathbf{G}$ & $\mathbf{T}$ & $\mathbf{O}$ \\
\hline 0 & 13 & 0 & 26 & 4 & 9 & 39 & 9 & 0 \\
0 & 17 & 0 & 28 & 3 & 10 & 38 & 3 & 0 \\
0 & 8 & 0 & 50 & 0 & 12 & 27 & 4 & 0 \\
0 & 14 & 8 & 32 & 3 & 5 & 32 & 5 & 0
\end{tabular}

$\mathrm{A}=$ macrophanérophytes $; \mathrm{M}=$ mésophanérophytes $; \mathrm{I}=$ microphanérophytes ; $\mathrm{N}=$ nanophanérophytes $; \mathrm{C}=$ chamaéphytes $; \mathrm{H}=$ hémicryptophytes ; $\mathrm{G}=$ géophytes $; \mathrm{T}=$ thérophytes $; \mathrm{O}=$ hydrophytes

et ainsi de suite de groupement en groupement. Enfin, elles sont chargées et traitées dans $\mathrm{SAS}^{\odot}$. Après lancement du logiciel, le chargement se fait par la procédure simple suivante $\{\mathrm{P} 1\}$ :

DATA TAB ;

INPUT A M I N C H G T O E S ;

CARDS ;

001300260409390900001

001700280310380300001

000800500012270400001

$\cdots$

Il faut prendre soin de donner les pourcentages avec deux chiffres significatifs suivis d'un blanc, ce qui permet de retrouver les neuf colonnes du tableau I. La dixième colonne, notée $\mathbf{S}$, indique le numéro du groupement. Toutes les données des divers groupements à comparer sont entrées à la suite. Une vérification est possible par la procédure $\{\mathrm{P} 2\}$ qui affiche le tableau constitué :

PROC PRINT DATA=TAB ;

RUN ;

Puis les spectres moyens des divers groupements sont donnés directement par la procédure $\{\mathrm{P} 3\}$ suivante :

PROC MEANS N MEAN FW=2 ;

BY S ;

RUN ;

MEANS calcule de nombreux paramètres caractéristiques des distributions individuelles : moyenne, variance, écart-type, dissymétrie..., mais les indications $\mathrm{N}$ et MEAN ont pour effet de ne retenir que les effectifs et les moyennes; la mention $\mathrm{FW}=2$ permet l'affichage avec seulement deux chiffres significatifs, ce qui suffit pour caractériser des profils biologiques (lorsqu'une moyenne est inférieure à 1 , la valeur affichée commence par un point, au stade de l'interprétation, il faut lire $0:$ zéro ou 1). La seconde ligne fait renouveler le calcul pour chaque groupement. La comparaison est obtenue par la procédure $\{\mathrm{P} 4\}$ :

PROC DISCRIM ALL ;

CLASS S ;

RUN ;

La mention ALL sur la première ligne indique qu'il s'agit de l'approche la plus générale de l'analyse discriminante et la seconde ligne indique que les numéros des groupements correspondent à la variable $\mathbf{S}$, la dernière. Enfin, comme les groupements traités ne comportent ni hydrophyte ni épiphyte, les variables correspondantes (O et $\mathrm{E})$ peuvent être négligées, ce qui est indiqué par l'introduction en ligne 3 de l'instruction «VAR A-T ; » qui applique $\{\mathrm{P} 4\}$ sur les variables $\mathrm{A}$ à $\mathrm{T}$ et exclut $\mathrm{O}$ et $\mathrm{E}$. 


\section{RESULTATS}

\section{Profils moyens}

La procédure $\{\mathrm{P} 3\}$ donne des résultats se présentant de la manière indiquée dans le tableau II avec, bien entendu, autant de sections du même type qu'il y a de valeurs de $\mathrm{S}$, donc de groupements en cours d'analyse. La flore de ce type de savane ne présente donc aucun macrophanérophyte, 13 p. 100 de mésophanérophytes, 2 p. 100 de microphanérophytes, 34 p. 100 de nanophanérophytes, etc.

\section{Tableau II}

Résultats obtenus par $\{$ P3 $\}$

\begin{tabular}{|c|c|c|}
\hline Variable & $\mathbf{N}$ & Mean \\
\hline A & 4 & 0 \\
\hline$M$ & 4 & 13 \\
\hline I & 4 & 2 \\
\hline $\mathrm{N}$ & 4 & 34 \\
\hline C & 4 & 3 \\
\hline $\mathrm{H}$ & 4 & 9 \\
\hline G & 4 & 34 \\
\hline $\mathrm{T}$ & 4 & 5 \\
\hline $\mathrm{O}$ & 4 & 0 \\
\hline$E$ & 4 & 0 \\
\hline
\end{tabular}

$\mathrm{S}=$ numéro du groupe $;$ variables = types biologiques $; \mathrm{N}=$ nombre de relevés $\mathrm{du}$ groupe $;$ Mean $=$ proportion moyenne du type correspondant dans le groupe

$\mathrm{A}=$ macrophanérophytes $; \mathrm{M}=$ mésophanérophytes $; \mathrm{I}=$ microphanérophytes ; $\mathrm{N}=$ nanophanérophytes $; \mathrm{C}=$ chamaéphytes $; \mathrm{H}=$ hémicryptophytes ;

$\mathrm{G}=$ géophytes $; \mathrm{T}=$ thérophytes $; \mathrm{O}=$ hydrophytes

Pour l'ensemble des cinq groupements de savane de la dition, les spectres biologiques moyens sont représentés dans les figures 1 à 4 et réunis dans le tableau III avec les nombres de relevés suivants :
$1: 10$
$2: 4$
$3: 7$
$4: 8$
$5: 4$

\section{Tableau III}

Profils moyens des cinq groupements de savane de la dition

\begin{tabular}{lrrrrr} 
Types & $\mathbf{1}$ & $\mathbf{2}$ & $\mathbf{3}$ & $\mathbf{4}$ & $\mathbf{5}$ \\
\hline A Macrophanérophytes & 0 & 0 & 0 & 1 & 0 \\
M Mésophanérophytes & 5 & 13 & 19 & 13 & 10 \\
I Microphanérophytes & 1 & 2 & 14 & 13 & 11 \\
N Nanophanérophytes & 14 & 34 & 52 & 41 & 21 \\
C Chamaéphytes & 4 & 3 & 3 & 2 & 2 \\
H Hémicryptophytes & 1 & 9 & 9 & 9 & 15 \\
G Géophytes & 70 & 34 & 2 & 11 & 41 \\
T Thérophytes & 5 & 5 & 1 & 10 & 0
\end{tabular}

A = macrophanérophytes $; \mathrm{M}=$ mésophanérophytes $; \mathrm{I}=$ microphanérophytes ; $\mathrm{N}=$ nanophanérophytes $; \mathrm{C}=$ chamaéphytes $; \mathrm{H}=$ hémicryptophytes ; $\mathrm{G}=$ géophytes ; $\mathrm{T}=$ thérophytes

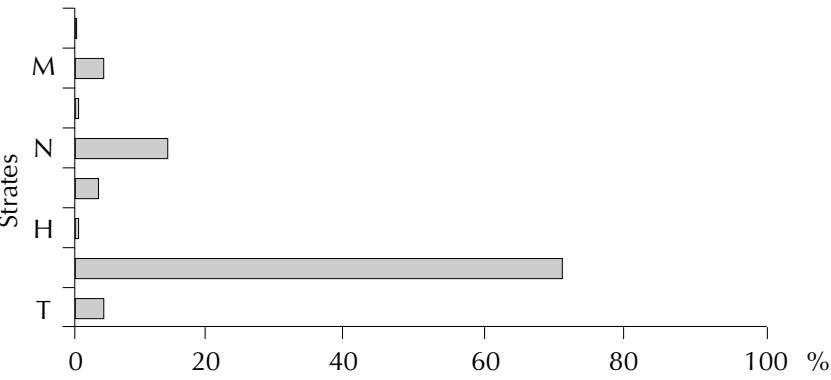

Figure 1 : spectre biologique moyen des savanes primaires arbustives - savane 1. M= mésophanérophytes ; $N=$ nanophanérophytes $; H=$ hémicryptophytes ; $T=$ thérophytes.

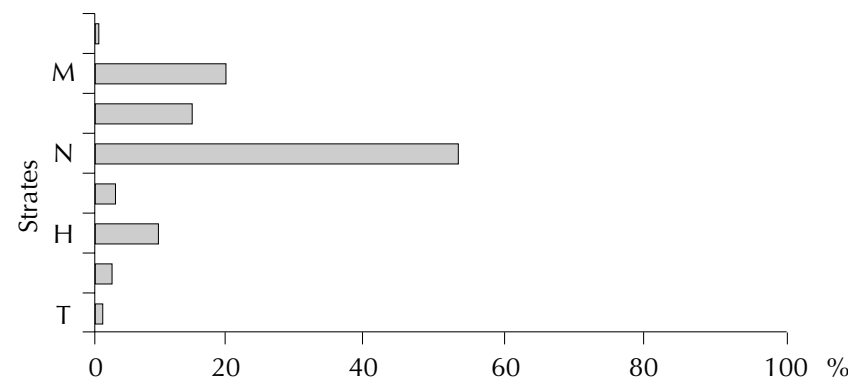

Figure 2 : spectre biologique moyen du groupement des savanes primaires à Cordyla pinnata - savane 3. $M=$ mésophanérophytes ; $\mathrm{N}=$ nanophanérophytes ; $\mathrm{H}=$ hémicryptophytes; $T=$ thérophytes.

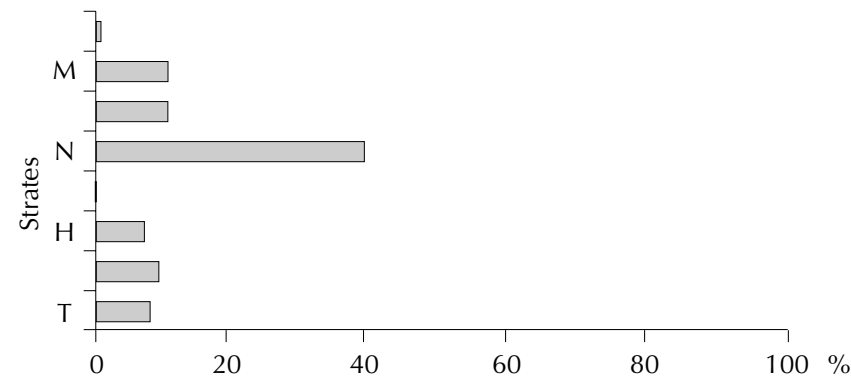

Figure 3 : spectre biologique moyen du groupement des savanes secondaires à Diospyros mespiliformis - savane 5. $M=$ mésophanérophytes $; N=$ nanophanérophytes $; H=$ hémicryptophytes ; $T=$ thérophytes.

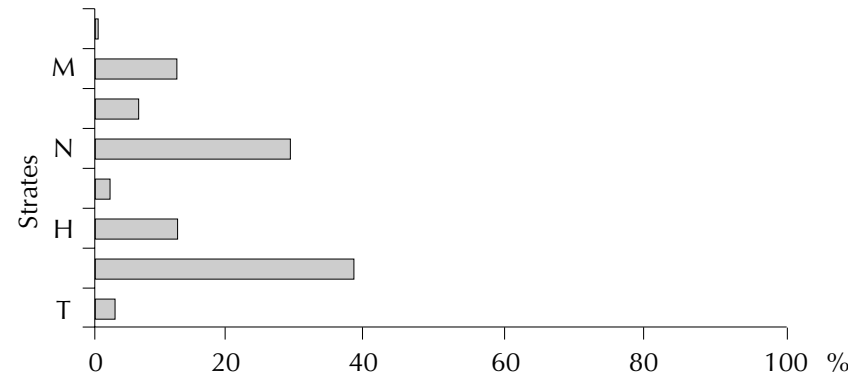

Figure 4 : spectre biologique moyen des groupements des savanes primaires à Ctenium newtonii et secondaires à Swartzia madagascarensis - savanes 2 et $4 . M=$ mésophanérophytes ; $N=$ nanophanérophytes ; $H=$ hémicryptophytes ; $T=$ thérophytes. 


\section{Comparaison des profils de savane}

La procédure $\{\mathrm{P} 4\}$ donne très rapidement une grande quantité de résultats répartis sur plusieurs pages. Il ne s'agit pas ici d'analyser les variables et leur " contribution », ni d'établir et d'étudier des " fonctions discriminantes », mais seulement de savoir si les groupements et leurs spectres moyens sont significativement différents. Les résultats pertinents sont réunis dans les tableaux IV et $\mathrm{V}$.

\section{Tableau IV}

Carrés des distances de Mahanalobis entre les spectres moyens

\begin{tabular}{rrrrrr} 
& \multicolumn{1}{c}{$\mathbf{1}$} & \multicolumn{1}{c}{$\mathbf{2}$} & \multicolumn{1}{c}{$\mathbf{3}$} & \multicolumn{1}{c}{$\mathbf{4}$} & \multicolumn{1}{c}{$\mathbf{5}$} \\
\hline 1 & 0,000 & 43,127 & 136,103 & 133,573 & 39,123 \\
2 & 43,127 & 0,000 & 42,593 & 43,479 & 9,189 \\
3 & 136,103 & 42,593 & 0,000 & 10,646 & 66,280 \\
4 & 133,573 & 43,479 & 10,646 & 0,000 & 71,506 \\
5 & 39,123 & 9,189 & 66,280 & 71,506 & 0,000
\end{tabular}

Le tableau IV donne les carrés des « distances de Mahanalobis» entre les spectres. La valeur de cette grandeur mesure l'écart entre les spectres biologiques moyens des différents groupements. Il apparaît que les spectres les plus éloignés sont 1 vs 3 et 4 , tandis que 2 et 5 comme 3 et 4 sont très proches.

\section{Tableau V}

Probabilités pour que les spectres moyens soient différents

\begin{tabular}{ccccc} 
& $\mathbf{2}$ & $\mathbf{3}$ & $\mathbf{4}$ & $\mathbf{5}$ \\
\hline 1 & 0,0001 & 0,0001 & 0,0001 & 0,0002 \\
2 & & 0,0002 & 0,0001 & 0,3393 \\
3 & & & 0,0346 & 0,0001 \\
4 & & & & 0,0001 \\
5 & & & &
\end{tabular}

Connaître l'écart entre spectres ne suffit pas pour savoir si, compte tenu du nombre de sites et de la dispersion au sein de chaque groupement, ces écarts sont significatifs ; la procédure $\{\mathrm{P} 4\}$ effectue ce test et les résultats figurent dans le tableau V. Deux hypothèses contradictoires sont en jeu :

- les spectres moyens des groupements $\mathbf{i}$ et $\mathbf{j}$ sont significativement différents aux seuils de $0,05,0,01$ et 0,001 (hypothèse $\mathrm{H} 0$ ) ;

- les spectres moyens ne sont pas significativement différents et peuvent donc être confondus (hypothèse $\mathrm{H} 1$ ).

Entre les spectres des groupements 1 et 2 la probabilité de 0,0001 est plus faible que le plus petit des trois seuils, donc l'hypothèse $\mathrm{H} 0$ peut être retenue et la différence entre leurs spectres biologiques est très significative ; entre 3 et $4, P=0,0346$, étant compris entre 0,05 et 0,01 , traduit une différence significative ; entre 2 et $5, P=0,339$ traduit une différence non significative qui conduit à rejeter $\mathrm{H} 0$ et à accepter H1. Dès lors, les données de ces deux groupements peuvent être réunies pour obtenir un seul spectre. En définitive, quatre spectres sont caractéristiques des savanes de la région.

\section{CONCLUSION}

L'étude des spectres biologiques est ancienne mais compliquée parce que chaque station a son propre spectre. Le problème des spectres caractéristiques d'associations ou de groupements végétaux est double. La première approche consiste à établir le spectre à partir de la totalité de la flore du groupement. La seconde à établir un spectre moyen à partir des divers spectres stationnels. C'est cette seconde approche qui est analysée ici sous la double perspective de l'établissement du spectre puis de la comparaison des spectres moyens. L'emploi de l'analyse discriminante constitue à nos yeux la méthode royale pour traiter ce sujet et nous proposons les moyens de l'aborder simplement par le biais du logiciel SAS ${ }^{\circledR}$.

\section{BIBLIOGRAPHIE}

1. ABERLIN J.P., 1986. Recherches phytosociologiques et phytogéographiques au Mali central. Thèse Doct., faculté Saint Jérome, Marseille, France, $544 \mathrm{p}$.

2. AUBREVILLE A., 1963. Classification des formes biologiques des plantes vasculaires en milieu tropical. Adansonia, 3 : 220-226.

3. BOUROCHE J.M., SAPORTA G., 1983. L'analyse des données. Paris, France, PUF, $172 \mathrm{p}$.

4. DAGNELIE P., 1975. Analyse statistique à plusieurs variables. Gembloux, Belgique, Presses agronomiques, $262 \mathrm{p}$.

5. EMBERGER L., 1966. Réflexions sur le spectre biologique de Raunkiaer. Bull. Soc. bot. France : 147-156. (Mémoires)

6. HELWIG J., 1987. Guide d'introduction au logiciel SAS de base. Cary, NC, USA, SAS Institute, $108 \mathrm{p}$.

7. HUMBOLDT A.D., 1807. Essai sur la géographie des plantes. Nanterre, France, Editions européennes Erasme, 155 p. + XXII + annexes.

8. LEBRUN J., 1964. A propos des formes biologiques en régions tropicales. Bull. Acad. R. Sci. Outre-Mer : 926-937.

9. LEBRUN J., 1966. Les formes biologiques dans les végétations tropicales. Bull. Soc. bot. France : 164-175. (Mémoires)

10. LEFEBVRE J., 1976. Introduction aux analyses statistiques multidimentionnelles. Paris, France, Masson, $220 \mathrm{p}$.

11. MUELLER-DOMBOIS D., ELLENBERG H., 1974. Aims and methods of vegetation ecology. New York, USA, J. Wiley, $547 \mathrm{p}$.

12. ORSHAN G., 1953. Note on the application of Raunkiaer's life forms in arid region. Palest. J. Bot., Jerusalem, 6: 120-122.

13. PAVILLARD J., 1935. Elements de sociologie végétale. Paris, France, Hermann, $102 \mathrm{p}$.

14. RAUNKIAER C., 1904. Om biologiske typer, med Hensyn til Planternes Tilpasning til at overle ugunstige Aarister. Bot. Tidsskrift, 26.

15. RAUNKIAER C., 1908. Livsformernes Statistik som Grundlag for Plantegeografi. Bot. Tidsskr., 29.

16. RAUNKIAER C., 1909. Formationsundersogelse og Formationsstatistik. Bot. Tidsskr., 30.

17. RAUNKIAER C., 1918. Recherches statistiques sur les formations végétales. Kgl. Dan. Vidensk. Selsk. Biol. Medd., 5.

18. SAUTORY O., 1995. La statistique descriptive avec le système SAS. Paris, France, Insee, $260 \mathrm{p}$.

19. TOMASSONE R., 1988. Comment interprèter les résultats d'une analyse factorielle discriminante. Boigneville, France, Itcf, $56 \mathrm{p}$.

20. TOMASSONE R., DANZART M., 1988. Discrimination et classement. Paris, France, Masson, 174 p.

Accepté le 26.02.2004 


\section{Summary}

Aberlin J.-P., Daget P. Establish and Compare the Life Form Spectra of Several Plant Groups

The structure of a site flora can be characterized by its life form spectrum which indicates each life form rate as defined by Raunkiaer for station flora. A group made of several stations can be characterized by an average life form spectrum. Some of the results of the discriminating analysis allow comparison of the life form spectra of several plant groups according to their internal variability: application to savannas of Mali.

Key words: Savanna - Measurement - Vegetation - Ecosystem Mali.

\section{Resumen}

Aberlin J.-P., Daget P. Establecer y comparar los espectros biológicos de varios grupos vegetales

La estructura de la flora de una estación puede ser caracterizada por su espectro biológico, que indica la tasa de cada uno de los tipos biológicos definidos por Raunkiaer para la flora estacional. Una agrupación, reuniendo varias estaciones puede caracterizarse por un espectro biológico medio. Ciertos resultados del análisis discriminante permiten la comparación de los perfiles biológicos de varios grupos vegetales, tomando en cuenta su variabilidad interna. Aplicación a grupos de sabanas de Mali.

Palabras clave: Sabana - Medicon - Vegetación - Ecosistema Malí. 